\title{
Razonamiento Quirúrgico en Inestabilidad Crónica Posterolateral de Rodilla
}

\section{Surgical Rationale in Chronic Posterolateral Knee Injury}

\author{
Pedro Alejandro Díaz Allende ${ }^{1}$ \\ ${ }^{1}$ Traumatólogo, Servicio Ortopedia y Traumatología Hospital \\ Ricardo Valenzuela Sáez, Rengo, Chile \\ Rev Chil Ortop Traumatol 2017;58:48-58.
}

\begin{abstract}
Address for correspondence Pedro Alejandro Díaz Allende, MD, Servicio Ortopedia y Traumatología Hospital Ricardo Valenzuela Sáez, Rengo, Chile (e-mail: drpedrodiaz@gmail.com).
\end{abstract}

\section{Resumen \\ Palabras Clave \\ - esquina posterolateral \\ - inestabilidad crónica \\ - osteotomía en doble nivel \\ - osteotomía tibial alta \\ - planificación preoperatoria \\ - rodilla}

Los pacientes con mal alineamiento en varo pueden ser difíciles de tratar cuando tienen una lesión crónica de la esquina posterolateral (EPL), resultando en doble o triple varo y subsecuentemente varus thrust durante la marcha. Una osteotomía tibial alta es un procedimiento útil y necesario particularmente en el estadío crónico. Este artículo revisa los fundamentos de la planificación preoperatoria en una lesión crónica de la EPL.
Abstract
Keywords
- chronic instability
- double level osteotomy
- high tibial osteotomy
- knee
- posterolateral corner
- preoperative planning

Patients with varus malalignment can be difficult to manage when they have a chronic injury to the posterolateral corner (PLC), resulting in double or triple varus and subsequent varus thrust during gait. A high tibial osteotomy is a useful and necessary procedure particularly in the chronic setting. This article reviews the basics of preoperative planning in a chronic injury of the PLC.

\section{Introducción}

La inestabilidad ligamentaria crónica de la esquina posterolateral (EPL) de rodilla es una indicación escasamente debatida para la realización de una osteotomía alrededor de la rodilla. De no considerarse como una alternativa primaria, puede conducir a una reconstrucción ligamentaria fallida y muchas veces innecesaria. La osteotomía tibial proximal permite corregir el alineamiento coronal y sagital, mejorando la función de una rodilla inestable con varus thrust durante la marcha. received

March 19, 2017

accepted

July 18, 2017

published online

August 23, 2017
DOI https://doi.org/

$10.1055 / \mathrm{s}-0037-1606127$. ISSN $0716-4548$.
Copyright (e) 2017 by Thieme Revinter

Publicações Ltda, Rio de Janeiro, Brazil
License terms

(요 (1) $\Theta \circledast$ 


\section{Importancia del Slope Tibial en el Manejo de la Insuficiencia Posterolateral}

Durante la marcha, la fuerza de contacto femorotibial (FT) se descompone en fuerzas compresivas y cizallantes. ${ }^{1} \mathrm{El}$ componente cizallante desplaza a la tibia anteriormente producto del slope tibial, lo que produce cambios en la tensión ligamentaria intra y extrarticular.

En la rodilla normal, el slope tibial fluctúa entre $6^{\circ}$ y $13^{\circ}$. En la literatura se describe un amplio rango de valores como normales por lo que es importante consignar qué técnica de medición es utilizada para su evaluación. Dejour y Boninn ${ }^{2}$ recomiendan trazar una línea perpendicular al eje tibial anatómico (que se corresponde con el 20\% anterior de la superficie articular tibial) y unirla con otra línea tangente a los puntos más altos (anterior y posterior), del platillo tibial medial (PTM). Moore y Harvey $^{3}$ recomiendan trazar la perpendicular a una tangente que pasa a lo largo de la corteza tibial anterior y unirla a la tangente del PTM. Brazier y col. $^{4}$ proponen 2 métodos adicionales. En el primero, se traza una línea a lo largo de la corteza tibial posterior y otra a lo largo del PTM. En la segunda, recomiendan trazar una línea a lo largo del eje anatómico fibular y otra a lo largo del PTM. De todas las técnicas, la propuesta por Dejour y Boninn es considerada la más confiable y reproducible ( - Fig. 1).

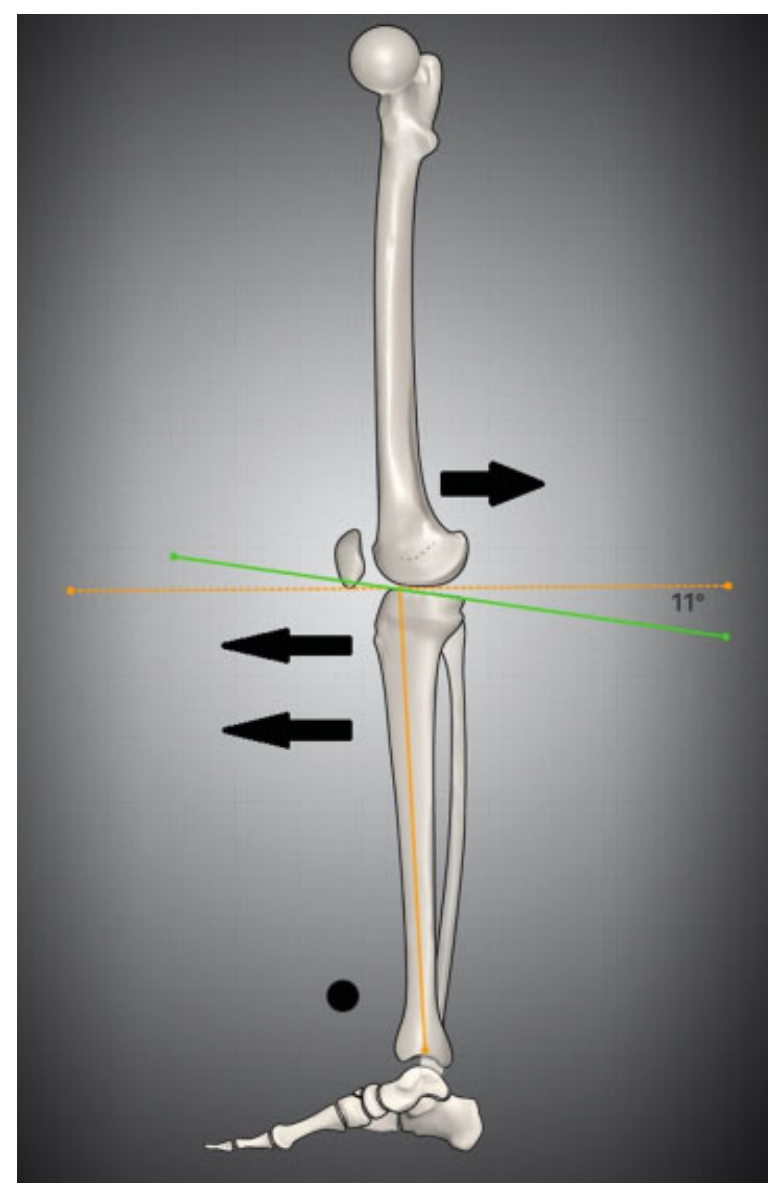

Fig. 1 Calculo Slope Tibial $\left(11^{\circ}\right)$ con técnica Dejour-Bonnin y traslacion tibial anterior producto de la inclinacion tibial posterior en el eje sagital.
En general, las osteotomías de tibia proximal en cuña cerrada lateral (OTPCCL), generan una disminución del slope tibial y traslación posterior de la tibia, lo que estabiliza las rodillas con insuficiencia del ligamento cruzado anterior (LCA), ${ }^{5}$ mientras que la osteotomía tibial proximal en cuña de apertura medial (OTPCAM), incrementa el slope tibial, provocando una traslación anterior de la tibia, y estabilizando insuficiencias del ligamento cruzado posterior (LCP) y EPL. ${ }^{6} \mathrm{~A}$ diferencia de la OTPCCL, la OTPCAM evita la desinserción muscular y lesión del nervio peroneo común, preserva la articulación tibiofibular proximal, no acorta la longitud de las estructuras posterolaterales y evita la osteotomía del peroné, lo que podría aumentar la inestabilidad de la EPL.

Griffin y col. ${ }^{6}$ en condiciones de laboratorio, concluyó que incrementando el slope posterior de $9^{\circ}$ a $13^{\circ}$, se permite que una fuerza axial de solo 200N (aproximadamente un cuarto del peso corporal), desplace anteriormente la tibia en $3 \mathrm{~mm}$. Liu-Barba y col, ${ }^{7}$ demostraron que con una fuerza de compresión de $1600 \mathrm{~N}$ (equivalente a 2 veces el peso corporal), la tibia se moviliza anteriormente $8 \mathrm{~mm}$ si el LCA está intacto y $13 \mathrm{~mm}$ si está seccionado. De esos estudios, se concluye que al incrementar el slope tibial, se da una traslación tibial anterior que estabiliza el LCP, tensa la EPL y reduce la hiperextensión, mejorando los síntomas del varus thrust. De manera inversa, con valores de slope tibial bajos o neutro existe una predisposición a elongar el LCP y la EPL.

Agneskirchner $\mathrm{y} \mathrm{col}^{8}$ demostraron en un estudio biomecánico que la subluxación tibial posterior, producto de una lesión in vitro del LCP, se neutraliza aumentando el slope en $5^{\circ}$ y se revierte (vale decir, se tensa el LCA) aumentándolo $5^{\circ}$ más. Así se concluye que pequeños cambios en el slope tienen poderosos efectos en la biomecánica de la articulación.

La posición de la placa a utilizar para fijar la osteotomía de apertura es otro punto crucial al momento de definir el slope tibial: una placa anteromedial incrementa el slope en promedio $6^{\circ}$, mientras que una situada en la mitad de la osteotomía no tiende a modificarlo. ${ }^{9}$ Noyes y col $^{10}$ calcularon el efecto del ángulo de apertura de una OTPCAM en el slope tibial, concluyendo que por cada incremento de $1 \mathrm{~mm}$ en el gap anterior, hay un aumento de $2^{\circ}$ en el slope. Petrigliano y $\mathrm{col}^{11}$ confirmaron que al incrementar el slope nativo en $5^{\circ}$, disminuye significativamente la traslación posterior en el compartimiento lateral $(5.8 \mathrm{~mm})$ y medial $(3.3 \mathrm{~mm})$ al realizar el test de cajón posterior. Marti y col $^{12}$ reportaron que cada $10^{\circ}$ de corrección del varo en una OTPCAM, existe un incremento promedio de $2,7^{\circ}$ en el slope tibial y una traslación tibial anterior de $6 \mathrm{~mm}$. Ese incremento disminuye el stress del LCP en un $56 \%$ con la rodilla en flexión de $30^{\circ}$ y en un $61 \%$ en flexión de $90^{\circ} .{ }^{13}$ Finalmente, existe un efecto condroprotector diferencial demostrado por Agneskirchner y $\mathrm{col}^{8} \mathrm{al}$ medir presiones de contacto después de incrementar el slope tibial, que consiste en la disminución de las fuerzas que actúan en la porción posterior de los platillos tibiales.

El objetivo de corrección del slope tibial, es incrementarlo entre $2^{\circ}$ y $8^{\circ}$ como límite superior, de modo de evitar el estrés sobre el LCA y el aumento de presión sobre el cartílago articular tibial anterior. ${ }^{8}$ 


\section{Fundamentos de la Planificación Preoperatoria en la Corrección del Varo}

El principio fundamental que permite una corrección adecuada del eje coronal es realizar la osteotomía en el segmento óseo que presenta la deformidad. Para ello es indispensable la medición angular adecuada del componente femoral distal, tibial proximal e intrarticular (-Fig. 2).

Se define como eje mecánico promedio en una telerradiografía, al ángulo entre el eje mecánico femoral y tibial a nivel de la rodilla. El eje mecánico femoral, es la línea trazada desde el centro de la cabeza femoral al centro del surco femoral. El eje mecánico tibial, se define como la línea desde el centro de las espinas tibiales y el punto medio de la mortaja tibial. La línea de carga de peso, se define como la línea trazada desde el centro de la cabeza femoral al centro de la mortaja tibial. Esa línea es importante, ya que permite realizar una relación puntual con otra línea trazada a lo largo del ancho de los platillos tibiales (donde el extremo más medial representa el 0\% y el más lateral el 100\% del ancho tibial), y así poder determinar el grado de corrección en la planificación de una osteotomía.

El ángulo mecánico lateral femoral distal (AMLFD), se define como el ángulo lateral que se forma de la conjunción de la línea tangente a los cóndilos femorales y el eje mecánico femoral. Su valor normal fluctúa entre $85^{\circ}-90^{\circ}$. El ángulo mecánico medial tibial proximal (AMMTP), se define como el

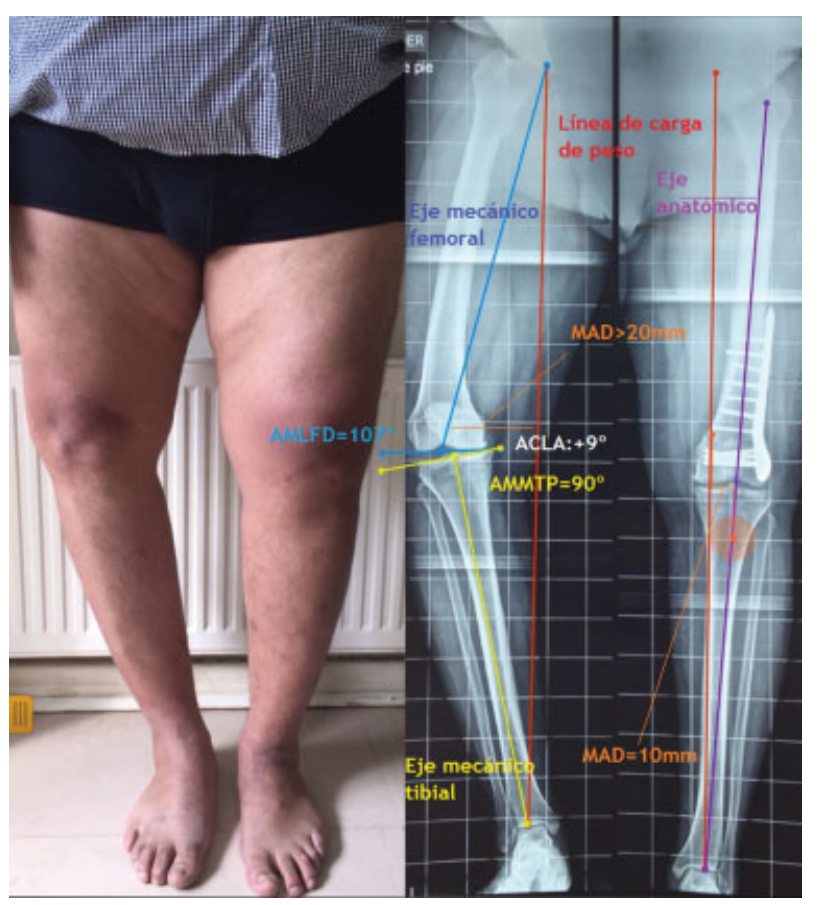

Fig. 2 Ejes y ángulos de la extremidad inferior. Paciente con osteotomía femoral valguizante rodilla izquierda y antecedente de genu varo severo bilateral. En espera de corrección quirúrgica a la derecha debido a varus thrust. Nótese la apertura del espacio articular lateral de la rodilla derecha por insuficiencia de la EPL. Abreviaciones: AMLFD, ángulo mecánico lateral femoral distal; AMMTP, ángulo mecánico medial tibial proximal; ACLA, ángulo de convergencia de la línea articular; MAD, desviación del eje mecánico (por sus siglas en inglés). ángulo medial entre la tangente a los márgenes de los platillos tibiales y el eje mecánico tibial. Su valor normal también fluctúa entre $85^{\circ}-90^{\circ}$. La alteración en esos valores determina dónde se encuentra la deformidad.

El ángulo de convergencia de la línea articular (ACLA), representa la deformidad intrarticular dada por la laxitud ligamentaria o la condropatía degenerativa y se define como el ángulo que conecta las líneas tangentes a las superficies articulares femoral y tibial. Se ha demostrado la influencia del ACLA en el alineamiento cuando se toman radiografías en posición de pie con carga. Su valor normal fluctúa entre $0^{\circ} \pm 2^{\circ}$. El estrechamiento medial genera un valor positivo del ACLA, contribuyendo al varo. Si el ápex del ACLA es lateral, genera un valor negativo, contribuyendo al valgo articular. Dudgale $\mathrm{y} \mathrm{col}^{14}$ demostró mediante fórmulas matemáticas, que por cada incremento de $1 \mathrm{~mm}$ en la apertura del espacio articular lateral, debido a la insuficiencia ligamentaria posterolateral, existe $1^{\circ}$ de varo agregado en las radiografías con carga, la cual debe sustraerse de la corrección del eje mecánico determinada en la planificación preoperatoria.

La estrecha relación entre el alineamiento de las extremidades inferiores y el ACLA puede ser en parte explicado por los cambios en el momento aductor del compartimiento medial. En alineamiento neutro, el momento aductor es insuficiente para abrir el espacio articular FT lateral debido a los restrictores externos: banda iliotibial y ligamento colateral lateral, haciendo el ACLA paralelo. En alineamiento varo, el momento aductor se incrementa debido a que el vector de la fuerza de reacción al peso durante la marcha sobrepasa el margen medial del platillo tibial. De esa forma, el momento aductor puede abrir el espacio articular lateral pese a los restrictores externos, que terminan por elongarse, incrementando el ACLA ${ }^{15}$ (-Fig. 2).

El resultado de una osteotomía dependerá fuertemente de una óptima planificación preoperatoria. Se debe evitar la recurrencia del varo por una corrección subestimada o artrosis del compartimiento femortotibial externo por sobre corrección. Se han propuesto múltiples fórmulas para conseguirlo, sin embargo, ninguna es perfecta. La mayoría de las recomendaciones se basan en el trabajo de Fujisawa y col $^{16}$ quien concluyó en 1979 que para óptimos resultados, el eje corregido debe pasar a través del 30-40\% del ancho del platillo tibial externo, tomando el punto de $0 \%$ en el centro de la rodilla. Basado en ese ensayo clínico, se recomienda por parte de muchos autores contemporáneos, que el eje mecánico debiese atravesar el platillo tibial externo, en un punto ubicado en el 62,5\% del ancho articular total de la tibia, tomando el $0 \%$ desde el extremo más medial de éste. ${ }^{14,17} \mathrm{Sin}$ embargo, el trabajo original de Fujisawa no provee una racionalidad biomecánica para usar ese punto, siendo más bien un juicio clínico.

Marti y col $^{12}$ intenta justificar el grado de corrección dependiendo del desgaste FT medial. Si no existe daño articular, la línea de carga de peso debiese pasar por el centro de la rodilla. Si se ha perdido un tercio del espesor condral, se debe desplazar al 55\% del ancho total del platillo tibial, desde su extremo medial al lateral. Con pérdidas de dos tercios del cartílago al 60\% y si existe perdida completa 
del espesor condral, al 65\%, sólo cubriendo las últimas 2 opciones el punto de Fujisawa.

Müller y Strecker, ${ }^{18}$ aseveran que la mejor forma de evidenciar el punto de sobrecorrección se basa en una artroscopía diagnóstica, con el fin de valorar el grado de lesión condral según la clasificación de Outerbridge ${ }^{19}$ en el mismo tiempo quirúrgico de la osteotomía (-Fig. 3). De ese modo, el punto de sobrecorrección debería ubicarse sobre el platillo tibial lateral con eje mecánico en $5^{\circ}$ de valgo para lesiones tipo IV (equivalente al 50\% del ancho del platillo tibial externo), en el punto de Fujisawa para lesiones tipo III ( $3,3^{\circ}$ de valgo mecánico, entre $30-40 \%$ de la superficie del platillo tibial lateral o $62,5 \%$ de la superficie articular tibial total ${ }^{14}$ ) y en $1,7^{\circ}$ de valgo mecánico para lesiones de grado II (equivalente a una distancia intermedia entre ambas espinas tibiales y el punto de Fujisawa). En rodillas sin daño condral, se sugiere ubicar el punto de corrección tibial entre ambas espinas tibiales $\left(1,2^{\circ}\right.$ de varo mecánico), equivalente a un eje mecánico normal. ${ }^{20-23}$

Agneskircher y $\operatorname{col}^{17}$ realiza hasta el momento el único estudio realizado bajo condiciones reproducibles de carga dinámica sobre la rodilla, midiendo la distribución de presión a través de la articulación antes y después de una osteotomía tibial valguizante, con el eje corregido a través del punto de Fujisawa, con un ligamento colateral medial superfcial (LCMs)

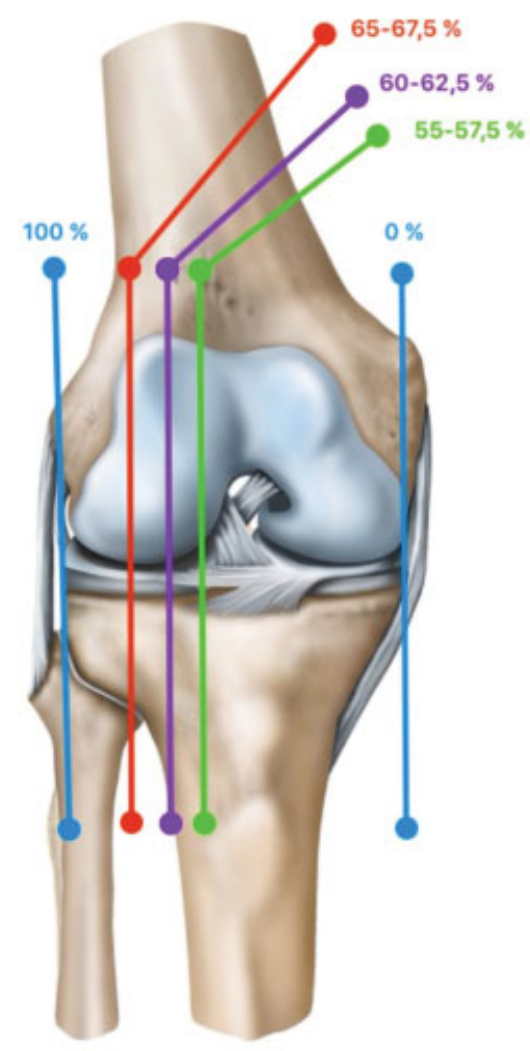

Fig. 3 Sobrecorrección del varo según el nivel de lesión condral en el compartimiento femorotibial (FT) medial (Outerbridge grado IV en rojo, Grado III en morado y Grado II en verde). Las líneas de colores corresponden al eje de carga de peso planificado a corregir en una osteotomía tibial alta, al intersectarse de forma puntual con el ancho del platillo tibial, donde las líneas azules representan los extremos medial $(0 \%)$ y lateral (100\%) de la superficie articular tibial. intacto y luego desinserto. En la rodilla en varo, la mayoría de la presión fue medial (65\%) al igual que en el alineamiento neutro (60\%). En alineamiento valgo la presión se traslada al compartimiento FT lateral (65\%). Después de la OTPCAM con LCMs intacto, paradójicamente la presión se incrementó medialmente (71\%) y solo después de la resección completa del LCMs la presión cambió hacia lateral (64\%). De esa forma, concluyeron que la descompresión del compartimiento medial es posible y efectiva usando una leve sobrecorrección en valgo, pero no sin antes liberar por completo el LCMs. Cuando el vector de carga pasó a través del centro de la rodilla, las presiones de contacto en el compartimiento lateral fueron un $70 \%$ más elevadas que en el compartimiento medial. Cuando ese vector pasó por el centro del compartimiento medial, las presiones de contacto se igualaron en ambos compartimientos. Eso es el reflejo de la geometría articular: el compartimiento lateral al ser menos congruente, presenta un área de contacto articular pequeño y por lo tanto, fuerzas compresivas locales generan incrementos desproporcionados en el estrés de carga articular. Eso debe tenerse en consideración al momento de planificar la descarga del compartimiento medial, por los posibles efectos deletéreos en su contraparte externa.

El objetivo de la corrección en condiciones condrales ideales, es realizar la osteotomía para trasladar el eje mecánico femoral y tibial al centro de la rodilla, lo que traduce un eje mecánico promedio de $0^{\circ} \pm 3^{\circ}$. Eso se acerca al eje mecánico normal de entre un $1,3^{\circ}$ de varo en la población con distribución Normal. ${ }^{20-23}$

\section{Consideraciones Respecto al Ángulo de Apertura o Cierre de la Osteotomía}

En este punto entran en juego otros 2 conceptos fundamentales descritos por Paley ${ }^{24}$ : la "desviación del eje mecánico" (MAD, por sus siglas en inglés) y el "centro de rotación de la angulación" (CORA, también por sus siglas en inglés). Además de 2 formulas derivadas de la trigonometría: la "tangente del ángulo de corrección" (que denominaremos "tangente de alfa") y el "teorema del coseno".

En términos simples, MAD es la desviación de la línea de carga de peso desde el centro de la rodilla. Fisiológicamente, un eje en población con distribución normal pasa $10 \pm 3 \mathrm{~mm}$ medial al centro de la articulación. El MAD ideal para la planificación debería estar en ese rango, siendo que $8 \mathrm{~mm}$ es el valor más cercano al eje mecánico normal $\left(1,2^{\circ}-1,3^{\circ}\right.$ de varo). Si la línea de carga de peso del paciente a corregir pasa de medial al MAD ideal, existe un mal alineamiento en varo, mientras que si pasa de lateral a éste, existe un mal alineamiento en valgo.

El punto de intersección de la proyección de la línea de carga de peso planificada con la cortical del segmento óseo a corregir, es conocido como CORA. Su importancia radica en que es el vértice del ángulo de corrección del mal alineamiento o punto donde rota la osteotomía. En condiciones ideales, ese punto debería encontrarse en una de las corticales metafisiarias de la tibia proximal o fémur distal, con el fin de propiciar un ambiente adecuado para la 


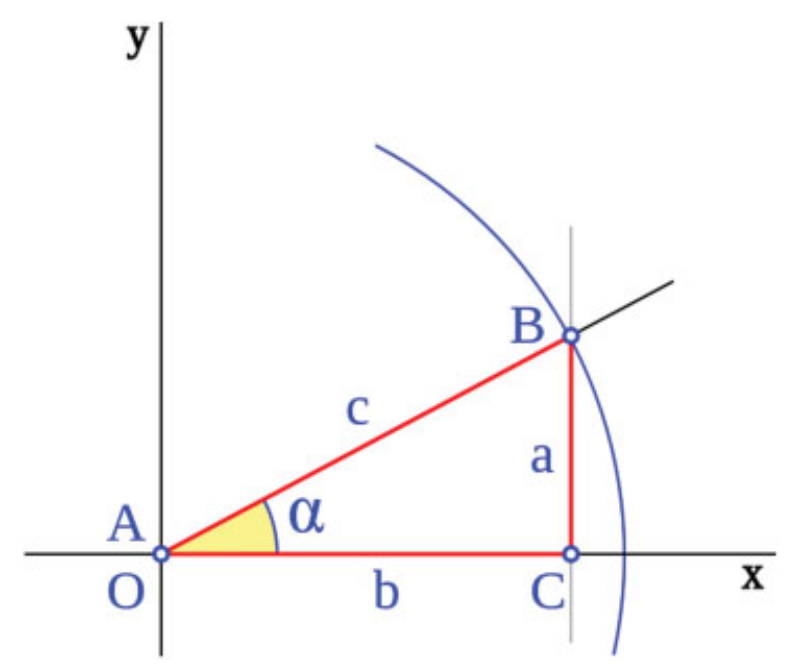

Fig. 4 Notación del Triángulo rectángulo.

consolidación ósea. La placa de fijación de la osteotomía (ya sea de apertura o cierre), se encuentra en la cortical opuesta al CORA.

Si consideramos que la osteotomía es la representación geométrica de un triángulo rectángulo ( - Fig. 4), la fórmula quedaría de la siguiente forma:

\section{Donde}

“ $\alpha$ ” (alfa) es el ángulo agudo que representa el ángulo de corrección.

"a" es el cateto opuesto al ángulo $\alpha$, que representa la apertura o cierre (en milímetros) de la osteotomía.

"b" es el cateto adyacente al ángulo $\alpha \mathrm{y}$ al ángulo recto, que representa el largo (en milímetros) de la línea de la osteotomía en el plano coronal desde una cortical a la opuesta.

Entonces, la razón entre el cateto opuesto y el adyacente determinan la "tangente de $\alpha$ " ( - Fig. 5).Si despejamos esa fórmula, podemos determinar de manera exacta los milímetros de apertura o cierre de la osteotomía, vale decir, el cateto opuesto.

$$
\operatorname{Tan} \alpha=\frac{a}{b} ; \text { Luego: } a=\tan \alpha_{\mathrm{x}} \mathrm{b}
$$

El Teorema del Coseno puede ser útil si el CORA no se ubica en un punto cortical donde se desea realizar la osteotomía. ${ }^{25} \mathrm{Con}$ esa fórmula, se puede elegir el lugar de la osteotomía independientemente de dónde se encuentre el CORA, y permite calcular por la proyección de los catetos, el ángulo exacto de corrección.

Para un triángulo cualquiera ( -Fig. 6) esa ecuación es válida si el lado "c" es adyacente a 2 ángulos agudos (triangulo acutángulo) o a 1 ángulo agudo y otro obtuso (triangulo obtusángulo).

\section{El Teorema se Describe Como:}

$$
c^{2}=a^{2}+b^{2}-2 a b \cos \gamma
$$

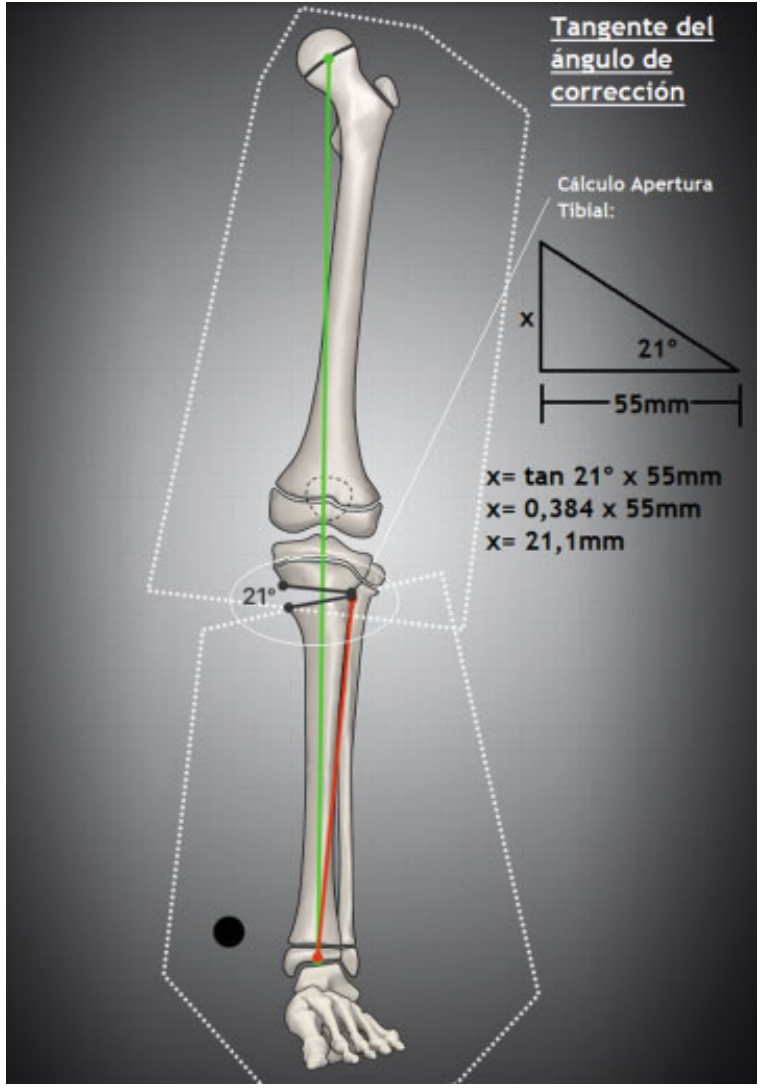

Fig. 5 Cálculo de la apertura (en milímetros), de una osteotomía tibial alta según la tangente del ángulo de corrección.

Esquemáticamente, se presenta un paciente sin lesión condral, por lo que se planifica una línea de carga de peso (en verde), que cruce el centro de la rodilla. La línea roja corresponde a aquella guía que traslada el fragmento óseo distal osteotomizado (con vértice en el CORA), desde el centro de la mortaja tibial de la línea de carga de peso original (no mostrada en la figura) hasta la línea de carga de peso planificada (en verde). Se desea realizar corte tibial en hueso metafisiario desde cortical medial hasta el CORA, midiendo $55 \mathrm{~mm}$ de largo. El ángulo alfa mide $21^{\circ}$. Según la formula descrita, se obtiene una apertura de $21,1 \mathrm{~mm}$.

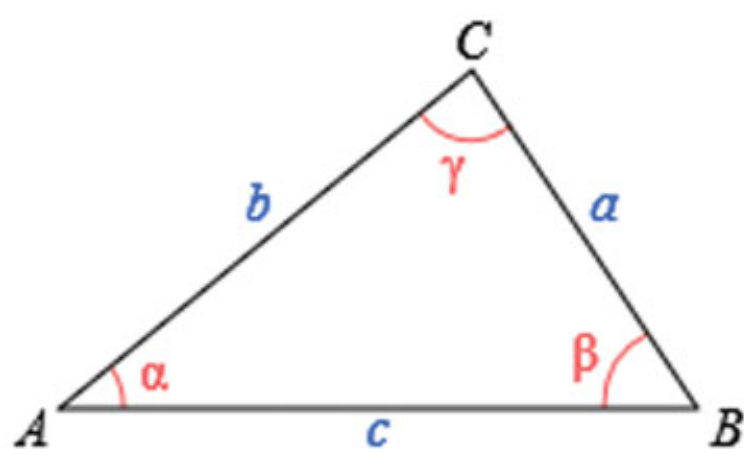

Fig. 6 Notación de Triángulo acutángulo. 


\section{Despejando la Formula se Obtiene el Ángulo de Corrección y, Donde}

$$
\gamma=\operatorname{arcos}\left(\frac{a^{2}+b^{2}-c^{2}}{2 a b}\right)
$$

"a" es el cateto adyacente al ángulo de corrección $\gamma$. Representa la distancia (en milímetros) entre el CORA planificado y la proyección de la línea de carga de peso planificada a la altura del centro de la mortaja tibial.

"b" es el otro cateto adyacente al ángulo de corrección $\gamma$. Representa la distancia (en milímetros) entre el CORA planificado y el centro de la mortaja tibial.

"c" es el cateto opuesto al ángulo de corrección $\gamma$. Representa la distancia (en milímetros) entre el centro de la mortaja tibial y la proyección de la línea de carga de peso planificada, a la altura del centro de la mortaja tibial.

Con esa fórmula, se logra obtener el ángulo de corrección de la osteotomía, con un CORA modificado a una zona ideal, evitando así ubicar la osteotomía en una zona diafisiaria que comprometa su consolidación (-Fig. 7).

Los 2 tipos de placas disponibles en Chile para realizar una osteotomía de apertura, basan su medición en el ángulo de apertura (Tomofix ${ }^{\circledR}$, Synthes $\mathrm{GmbH}$, Oberdorf, Suiza), o en el cateto opuesto a ese ángulo, en milímetros de apertura (Placa Puddu ${ }^{\circledR}$, Arthex, Naples, USA).

Debe considerarse una simplificación a esos cálculos (tras el planteamiento trigonométrico planteado), el aproximar $1^{\circ}$ de apertura a $1 \mathrm{~mm}$ de apertura, ya que al utilizar esa regla se subestima el valor de corrección.

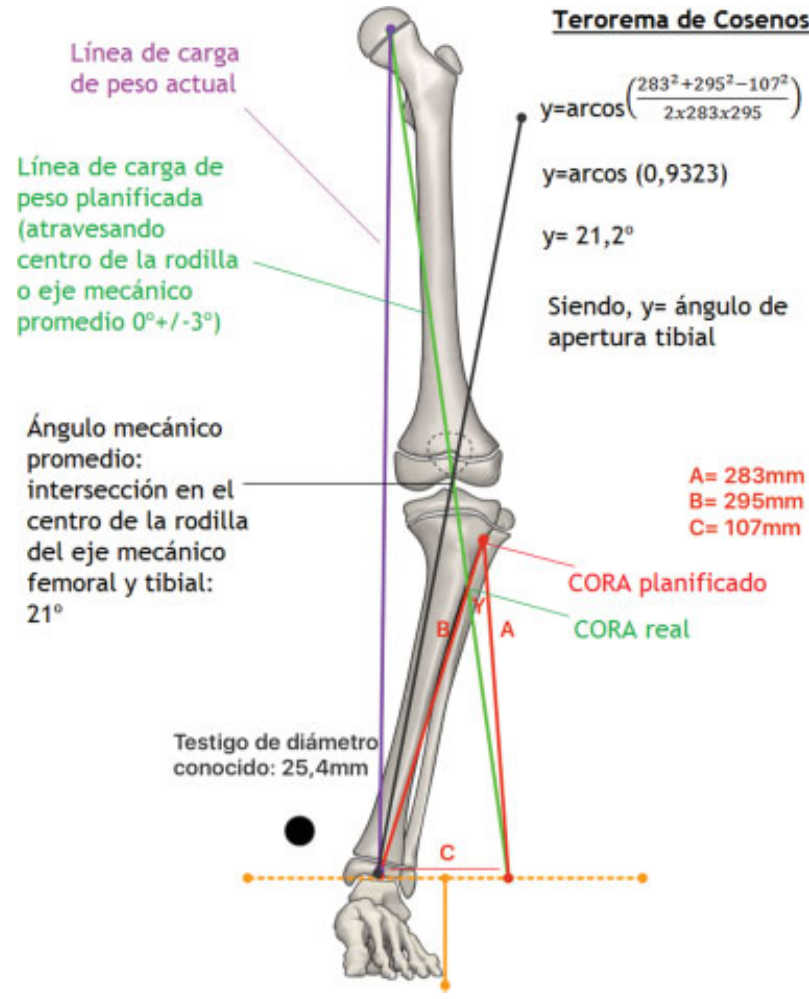

Fig. 7 Determinación del CORA planificado y ángulo de apertura de la osteotomía (se comprueba el razonamiento trigonométrico planteado en la revisión).
Para todas las mediciones planteadas hasta el momento, es necesario que la telerradiografía cumpla con 2 requisitos. El primero, es que se encuentre visible la escala a la cual está reducida la imagen, ya que los cálculos deben transformarse a una escala real del $100 \%$. El segundo, se basa en conocer la medida exacta de un componente de la imagen, que comúnmente puede ser el tamaño de la cuadrícula, una regla o un testigo de tamaño universalmente conocido (ej: moneda), con el fin de calibrar las mediciones.

\section{Consideraciones Respecto a la Altura Patelar}

En cuanto a la modificación de la altura patelar producto de una OTPCAM, es importante medir el índice CatonDeschamps (ICD) y evaluar el tamaño de la cuña de apertura. El ICD se obtiene en una radiografía lateral de rodilla en $20^{\circ}$ de flexión y se define como la relación entre la distancia desde el punto más distal de la superficie articular patelar y el borde anterosuperior de la tibia (numerador), por el largo de la superficie articular de la patela (denominador). Generalmente, la consecuencia no deseada de esa corrección es el desarrollo de una patela baja (ICD $\leq 0.6$ ), que limita el rango de flexión y promueve el desarrollo de artrosis patelofemoral al cambiar el área de contacto proximalmente.

Gaasbeek y col $^{26}$ estudió los efectos de la altura patelar tras una osteotomía estándar y otra modificada, en la que se realiza un corte adicional en un plano oblicuo (en "V" invertida) con $135^{\circ}$ de angulación respecto a la osteotomía clásica, a nivel de la tuberosidad anterior de la tibia (TAT), lo que permite mantenerla unida al fragmento tibial proximal (-Fig. 8). Demostró que la altura patelar no se altera al realizar la osteotomía modificada. Sin embargo, en una osteotomía estándar ésta disminuye significativamente. Mientras mayor es el ángulo de corrección, mayor será el descenso patelar.

Es importante mencionar que el dolor patelofemoral no es una contraindicación para realizar una OTPCAM, teniendo en consideración que la traslación anterior de la tibia que ésta produce al aumentar el slope, genera una disminución de la tensión del tendón patelar, mejorando los síntomas de una artrosis patelofemoral ${ }^{27}$ al disminuir la presión de la faceta lateral y el tilt lateral en promedio $2^{\circ}$.

Las osteotomías biplanares tibiales se han ido popularizando en parte debido a que las superficies óseas del corte vertical permanecen en estrecho contacto en un plano coronal detrás de la TAT, lo que promueve la consolidación. También tenemos los beneficios que provoca esta configuración de corte en la estabilidad rotacional, si la estabilización de ésta se realiza con una placa de diseño corto (tipo Puddu). Pape y $\operatorname{col}^{28} \sin$ embargo, concluye que si se utilizan placas más largas o bloqueadas, esa ventaja biomecánica no es estadísticamente significativa en relación a un corte uniplanar.

Basado en esos resultados, se establece que la osteotomía modificada debería realizarse en todo paciente que requiere 


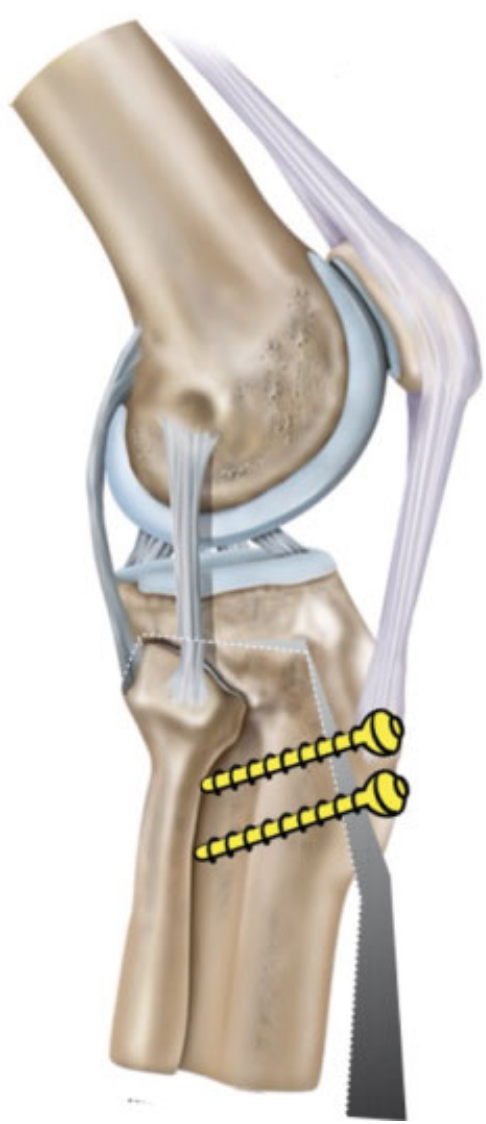

Fig. 8 Visión lateral de la osteotomía biplanar en "V." La placa de apertura tibial se posiciona por medial.

una corrección mayor de 8 a $10^{\circ}$ o con patela baja preexistente.

\section{Manejo de la Oblicuidad de la Línea Articular}

Algo que difícilmente se puede encontrar descrito en la literatura es cómo manejar la oblicuidad de la línea articular en caso que la deformidad ósea sea lo suficientemente significativa como para evitar su normalización tras una osteotomía o que la cuña de apertura planificada sea demasiado grande, comprometiendo su fijación y consolidación.

La inclinación medial de la línea articular genera fuerzas que subluxan la tibia hacia el lateral, sobretensando las estructuras estabilizadoras posterolaterales. Una osteotomía en doble nivel, es una combinación de osteotomías correctivas en la porción proximal de la tibia y distal del fémur. El procedimiento fue introducido por Benjamin $^{29}$ en 1969 para aliviar el dolor como manejo primario en pacientes con artritis reumatoide y artrosis que no respondieron al tratamiento conservador, sin embargo, no describió ninguna racionalidad biomecánica para justificarlo. Posteriormente, existieron estudios que fallaron en probar la validez de las sugerencias de Benjamin, ${ }^{30,31}$ por lo que fue olvidado por varias décadas. No es hasta el año 2002, que Babis y $\operatorname{col}^{32}$ realizó un estudio prospectivo para evaluar el concepto original y validar biomecánicamente las ventajas del procedimiento, identificando los individuos que se pueden beneficiar de éste. Es así como concluyó que la doble osteotomía es un procedimiento indicado en aquellos pacientes con genu varo severo (eje mecánico promedio $>8^{\circ}$ ), en los que no puede lograrse una oblicuidad articular aceptable $\left(0^{\circ} \pm 4^{\circ}\right)$ con una osteotomía en un nivel.

Saragaglia y col $^{33-35}$ es el único autor con publicaciones que describen la experiencia en osteotomías en doble nivel con un tamaño muestral significativo. En sus trabajos, define las indicaciones para realizar este procedimiento en genu varo severo, mediante una osteotomía femoral distal en cuña cerrada lateral (OFDCCL) y una OTPCAM. Eso lo realiza mediante navegación intraoperatoria con sistema Orthopilot ® (B-Braun-Aesculap, Tuttlingen, Alemania), placa bloqueada de fémur distal y placa Puddu ${ }^{\circledR}$ a tibia proximal. Las indicaciones consisten en: genu varo mecánico severo $>8^{\circ}$, artrosis Ahlback $<\mathrm{IV}$ (estrechamiento del espacio articular sin colapso óseo) y edad $<65$ años. Los objetivos de corrección: eje mecánico promedio entre $0^{\circ}-4^{\circ}$ de valgo, AMLFD $87^{\circ} \pm 2^{\circ} \mathrm{y}$ AMMTP $90^{\circ} \pm 2^{\circ}$. Los autores ${ }^{33-35}$ prefieren no sobrecorregir el eje mecánico argumentando principalmente las dificultades que eso puede producir en una revisión hacia una artroplastia total de rodilla. Otra ventaja de ese procedimiento, es la disponibilidad inmediata de autoinjerto estructural para la cuña de apertura tibial, proveniente de la cuña de sustracción femoral, evitando el alto costo de los aloinjertos o la morbilidad asociada del sitio donante en autoinjertos.

\section{Nuestro Enfrentamiento}

En nuestro Hospital, se solicitan radiografías de rodilla AP con carga, lateral y axial de rótulas. Con ellas se obtiene una imagen general de la sobrecarga compartimental, slope tibial y altura patelar. La telerradiografía de extremidades inferiores es el estudio preoperatorio fundamental. La resonancia nuclear magnética de rodilla confirma lesiones asociadas (meniscales y del pivote central principalmente).

Generalmente, para la planificación de ese tipo de osteotomías es necesario contar con un programa computacional que provea las mediciones angulares obtenidas de los ejes calculados en las telerradiografías, y que sea preciso en la evaluación de múltiples opciones quirúrgicas, incluyendo osteotomías de apertura y cierre, con sus resultantes ángulos femorotibiales, oblicuidad de la línea articular y acortamientoalargamiento de las extremidades con esos cambios. Todo ello en vista de no contar con un sistema de navegación computacional en la práctica quirúrgica habitual.

El programa para portátiles Bone Ninja ${ }^{\circledR}$ (desarrollado por los Dres. Shawn Standard y John Herzenbergel en el International Center for Limb Lengthening: Rubin Institute for Advanced Orthopedics. Sinai Hospital, Baltimore), es de extrema utilidad, logrando planificaciones realistas y cálculos que, de otra forma, requerirían las operaciones trigonométricas descritas previamente (-Fig. 9).

La cirugía se inicia con una artroscopia de rodilla, que confirme el grado de corrección planificado según el daño 


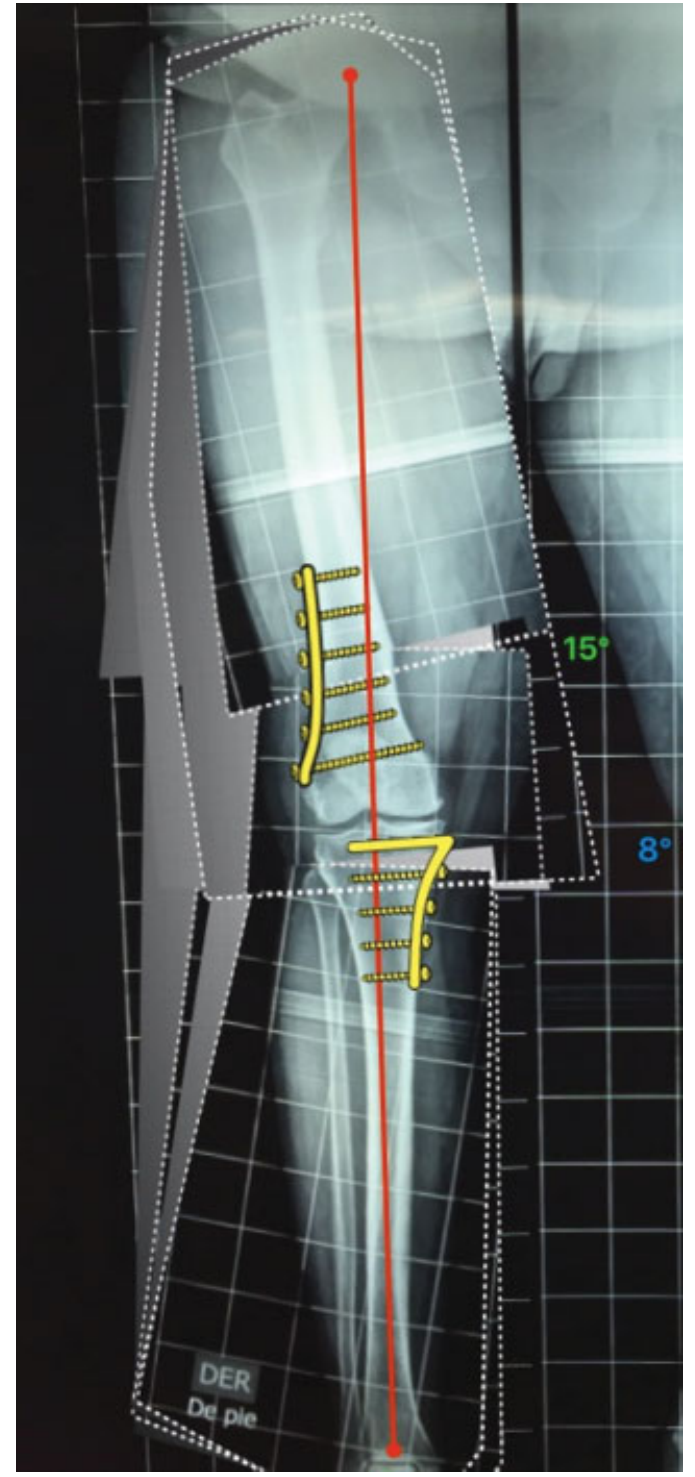

Fig. 9 Planificación de una Osteotomía en Doble Nivel. En verde, ángulo de la cuña de cierre femoral $\left(15^{\circ}\right)$. En azul, ángulo de la cuña de apertura tibial $\left(8^{\circ}\right)$. El paciente en cuestión se muestra en $\boldsymbol{- F i g . ~} 2$.

condral. La osteotomía inicial será femoral en caso de requerir la corrección de un varo severo con oblicuidad de la línea articular, ante una apertura tibial aislada valorada como excesiva en la planificación (que comprometa su estabilidad o consolidación), o en caso de que tengamos un AMLFD $>90^{\circ}$. Se asegura un constructo más estable mediante una OFDCCL con placa bloqueada anatómica.

El tipo de OTPCAM dependerá de la altura patelar, según lo descrito previamente. ${ }^{26}$ Se utiliza el autoinjerto extraído de la cuña femoral para sustentar la apertura tibial ( - Fig. 10).

De no requerir osteotomía femoral, preferimos el autoinjerto de la cresta iliaca por su valor osteogénico y osteoinductor, asociado al bajo costo. La fijación la realizamos con placa Puddu de acero, considerando que es una zona metafisiaria y apoyada con injerto óseo.

Es de vital importancia evitar el uso de mango de isquemia debido al tiempo que requiere la realización total de ese procedimiento. Un riesgo grave en esos casos, es la
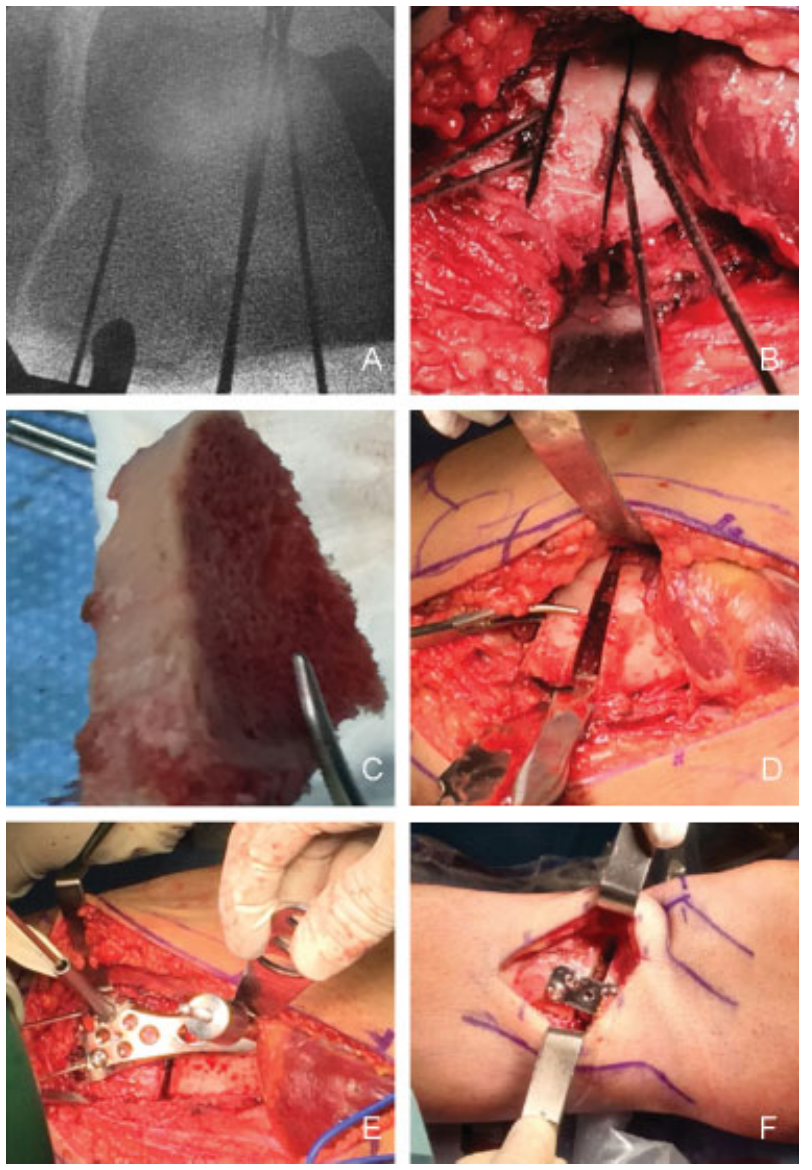

Fig. 10 Proceso quirúrgico en una doble osteotomía. A, B y C. Se inicia procedimiento con una osteotomía femoral distal en cuña de cierre lateral (OFDCCL) según planificación preoperatoria. D. Cuña de resección femoral, que será utilizada como autoinjerto. E. Fijación femoral con placa bloqueada. F. Osteotomía tibial proximal en cuña de apertura medial (OTPCAM) con Placa Puddu e injerto óseo proveniente de cuña de cierre femoral.

rabdomiolisis, ${ }^{36,37}$ que puede conducir a una falla renal aguda y necesitar de hemodiálisis transitoria en los casos más severos. Actualmente, para evitar esa complicación, utilizamos ácido tranexámico en dosis endovenosa de $10 \mathrm{mg} / \mathrm{kg}, 15$ minutos previos a la cirugía y de requerirlo, 1 dosis de refuerzo durante el intraoperatorio con el fin de reducir las pérdidas hemáticas, ${ }^{38-40}$ mejorando así la visualización de los tejidos. Con ese protocolo, reducimos al mínimo el uso de mango de isquemia en procedimientos quirúrgicos prolongados.

\section{Discusión}

Desde el punto de vista imagenológico, el estudio más importante es la telerradiografía de extremidades inferiores, ya que permite la planificación preoperatoria. El reflejo de una imagen adecuada se basa en la orientación anterior de las rótulas. Cualquier rotación debe ser corregida, teniendo en consideración que en esta patología existe una rotación tibial externa excesiva que puede alterar su posición.

Las radiografías dinámicas con estrés en el plano coronal y sagital han demostrado utilidad en lesiones agudas ${ }^{41}$ al igual 
que la resonancia nuclear magnética. ${ }^{42,43}$ En lesiones crónicas, su valor en la toma de decisiones es más discutible.

Respecto al tratamiento de la inestabilidad crónica de la EPL, la indicación primaria es la osteotomía. ${ }^{13,44-46}$ Está demostrado que el alineamiento de la extremidad inferior es un potente determinante del momento aductor con la correspondiente carga tensil del compartimiento FT externo. Se debe tener precaución respecto de los potentes efectos que genera una alteración de la inclinación del platillo tibial tanto en el plano coronal como en el sagital. El error más común de apreciar en el plano coronal es realizar una sobrecorrección, mientras que en el plano sagital es generar patela baja y déficit de extensión. ${ }^{47}$

La reconstrucción ligamentaria sin corrección del alineamiento, conduce a malos resultados clínicos a consecuencia de la sobretensión de partes blandas por la deformidad ósea. ${ }^{44,45}$ Naudine y col $^{48}$ en un estudio clínico con seguimiento a 56 meses confirma esa aseveración: tras el manejo de la inestabilidad crónica posterolateral con OTPCAM, solo el $30 \%$ de los pacientes requirió una reconstrucción ligamentaria en un segundo tiempo quirúrgico por persistencia de inestabilidad.

En general, si la osteotomía es insuficiente para generar estabilidad clínica tras 6-8 meses de evolución, se debe considerar una reconstrucción anatómica de los componentes ligamentarios insuficientes. ${ }^{44,45,49}$ Noyes y col $^{46}$ afirman que la inestabilidad persiste tras una osteotomía, en los casos en que la insuficiencia posterolateral permita una apertura del espacio FT lateral $\geq 12 \mathrm{~mm}$.

El nivel de la osteotomía se basa en determinar, mediante mediciones angulares, dónde se encuentra la deformidad primaria. En la tibia, se recomienda una OTPCAM, ya que ellas permiten aumentar el slope tibial, siendo útiles en el tratamiento de ese tipo específico de inestabilidad. En el fémur, se recomienda una OFDCCL debido a que se desea asegurar una consolidación adecuada y rehabilitación precoz.

Si la planificación preoperatoria no logra resolver algún problema en la oblicuidad de la línea articular, se recomienda realizar la osteotomía en dos niveles (-Fig. 11), procedimiento que requerirá contar con la asistencia de algún programa computacional para la planificación preoperatoria más precisa.

La artroscopia debería realizarse rutinariamente para confirmar la necesidad de sobrecorregir un genu varo asociado a la artrosis. En ausencia de una mejor evidencia, la recomendación principal es que de no existir condropatía asociada, la línea de carga de peso debería atravesar el centro de la rodilla. Eso probablemente sea una simplificación que muestra que no existe un punto específico aplicable a todos los pacientes. La respuesta a esa disquisición, probablemente se obtenga en el futuro con estudios enfocados en el análisis dinámico de la marcha de los pacientes afectados y sus peculiares adaptaciones individuales que ayudan a reducir su incomodidad.

En el acto quirúrgico, se debe liberar el ligamento colateral medial superficial a nivel de la osteotomía tibial valguizante, para permitir una real descarga del compartimiento medial. De lo contrario, se está aumentando la presión local por sobretensión del LCMs, lo que puede empeorar los síntomas y la progresión del daño articular. ${ }^{17}$

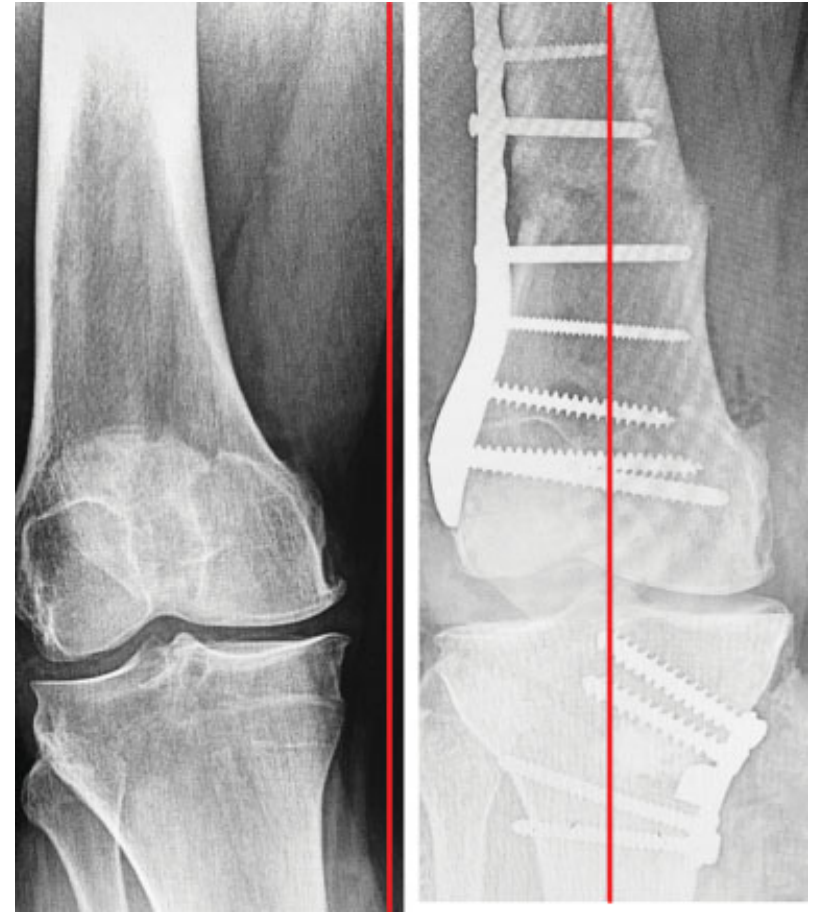

Fig. 11 Antes y después de la doble osteotomía. En rojo, el eje de carga de peso pre y postoperatorio.

La ventaja teórica de la osteotomía sobre las partes blandas en el plano coronal sería el retensado de la capsula articular posterior y del ligamento poplíteo oblicuo, ${ }^{50}$ los cuales pueden, en última instancia, fomentar una estabilidad posterolateral adicional. ${ }^{44,45}$

La posición de la cuña de apertura es muy importante en el sentido de corregir la deformidad en el plano sagital. El incremento del slope tibial ha demostrado ser la corrección más importante desde el punto de vista ligamentario en esa patología, ya que contrarresta la insuficiencia de las partes blandas al generar traslación tibial anterior, mejorando los síntomas del varus thrust. ${ }^{48}$

Existe escasa literatura al respecto de los efectos del slope tibial en la estabilidad rotacional. Petrigliano y $\mathrm{col}^{11}$ concluyen que al aumentar el slope en $5^{\circ}$ no existen diferencias significativas en la magnitud de la rotación tibial al realizar test clínicos (Dial test y Pivot Shift Reverso), respecto a una rodilla insuficiente sin osteotomía. Sin embargo, la disminución del slope en $5^{\circ}$ genera aumentos significativos de la inestabilidad rotacional de la rodilla al realizar los mismos test clínicos. Los autores hipotetizan que debe existir un límite de aumento del slope que se tiene que superar para encontrar una reducción significativa de la inestabilidad rotacional y éste debe ser $>5^{\circ}$.

\section{Conclusiones}

Mientras se evidencian buenos resultados tras la reconstrucción aguda de la esquina posterolateral, la literatura demuestra pobres resultados de procedimientos aislados sobre partes blandas en inestabilidad crónica, 
debido a las fuerzas de tensión excesivas sobre esas estructuras a consecuencia del mal alineamiento progresivo.

La evaluación ósea en el plano coronal y sagital es fundamental para la planificación preoperatoria. El rol de la osteotomía se basa en prevenir la progresión del deterioro unicompartimental, proteger las partes blandas de las elevadas cargas tensiles y restablecer la estabilidad clínica de la marcha al disminuir el momento aductor y recurvatum.

La literatura disponible es escasa y heterogénea respecto a las indicaciones y resultados funcionales al largo plazo.

\section{Agradecimientos}

A los Doctores: David Figueroa P., Rafael Calvo R. y Carlos Stierling D. de Clínica Alemana de Santiago. A los Doctores: Carlos Bolomey E., Javier Cayazaya D. y Pedro Gómez C. del Hospital Clínico FUSAT de Rancagua, por sus valiosos aportes en esta revisión, por incentivar el espíritu crítico y el rigor científico basado en la evidencia. A todo el equipo de Traumatología del Hospital Ricardo Valenzuela Sáez de Rengo por el apoyo mostrado en la realización de este artículo: Doctores Julio Hernández E., Joel Hernández DL., Alexander Pérez A., Francisco Neumann C., Mario Humeres R. y Patricia Salazar M.

\section{Conflicto de Intereses}

La realización de este estudio no cuenta con conflicto de intereses por parte del autor.

\section{Declaración y Verificación de la Presentación}

Este trabajo no ha sido previamente publicado ni presentado simultáneamente en otra revista y tiene la autorización del autor y su establecimiento asistencial.

\section{Bibliografía}

1 Amis AA. Biomechanics of high tibial osteotomy. Knee Surg Sports Traumatol Arthrosc 2013;21(01):197-205

2 Dejour H, Bonnin M. Tibial translation after anterior cruciate ligament rupture. Two radiological tests compared. J Bone Joint Surg Br 1994;76(05):745-749

3 Moore TM, Harvey JP Jr. Roentgenographic measurement of tibial-plateau depression due to fracture. J Bone Joint Surg Am 1974;56(01):155-160

4 Brazier J, Migaud H, Gougeon F, Cotten A, Fontaine C, Duquennoy A. [Evaluation des méthodes de mesure radiographique de la pente tibiale. Analyse de 83 genoux témoins]. Rev Chir Orthop Repar Appar Mot 1996;82(03):195-200

5 Hohmann E, Bryant A, Imhoff AB. The effect of closed wedge high tibial osteotomy on tibial slope: a radiographic study. Knee Surg Sports Traumatol Arthrosc 2006;14(05):454-459

6 Giffin JR, Stabile KJ, Zantop T, Vogrin TM, Woo SL, Harner CD. Importance of tibial slope for stability of the posterior cruciate ligament deficient knee. Am J Sports Med 2007;35(09):1443-1449

7 Liu-Barba D, Hull ML, Howell SM. Coupled motions under compressive load in intact and ACL-deficient knees: a cadaveric study. J Biomech Eng 2007;129(06):818-824

8 Agneskirchner JD, Hurschler C, Stukenborg-Colsman C, Imhoff $A B$, Lobenhoffer P. Effect of high tibial flexion osteotomy on cartilage pressure and joint kinematics: a biomechanical study in human cadaveric knees. Winner of the AGA-DonJoy Award 2004. Arch Orthop Trauma Surg 2004;124(09):575-584
9 Rodner CM, Adams DJ, Diaz-Doran V, et al. Medial opening wedge tibial osteotomy and the sagittal plane: the effect of increasing tibial slope on tibiofemoral contact pressure. Am J Sports Med 2006;34(09):1431-1441

10 Noyes FR, Goebel SX, West J. Opening wedge tibial osteotomy: the 3-triangle method to correct axial alignment and tibial slope. Am J Sports Med 2005;33(03):378-387

11 Petrigliano FA, Suero EM, Voos JE, Pearle AD, Allen AA. The effect of proximal tibial slope on dynamic stability testing of the posterior cruciate ligament- and posterolateral corner-deficient knee. Am J Sports Med 2012;40(06):1322-1328

12 Marti CB, Gautier E, Wachtl SW, Jakob RP. Accuracy of frontal and sagittal plane correction in open-wedge high tibial osteotomy. Arthroscopy 2004;20(04):366-372

13 Giffin JR, Vogrin TM, Zantop T, Woo SL, Harner CD. Effects of increasing tibial slope on the biomechanics of the knee. Am J Sports Med 2004;32(02):376-382

14 Dugdale TW, Noyes FR, Styer D. Preoperative planning for high tibial osteotomy. The effect of lateral tibiofemoral separation and tibiofemoral length. Clin Orthop Relat Res 1992;(274): 248-264

15 Lee DH, Park SC, Park HJ, Han SB. Effect of soft tissue laxity of the knee joint on limb alignment correction in open-wedge high tibial osteotomy. Knee Surg Sports Traumatol Arthrosc 2016;24 (12):3704-3712

16 Fujisawa Y, Masuhara K, Shiomi S. The effect of high tibial osteotomy on osteoarthritis of the knee. An arthroscopic study of 54 knee joints. Orthop Clin North Am 1979;10(03): 585-608

17 Agneskirchner JD, Hurschler C, Wrann CD, Lobenhoffer P. The effects of valgus medial opening wedge high tibial osteotomy on articular cartilage pressure of the knee: a biomechanical study. Arthroscopy 2007;23(08):852-861

18 Müller M, Strecker W. Arthroscopy prior to osteotomy around the knee? Arch Orthop Trauma Surg 2008;128(11):1217-1221

19 Outerbridge RE. The etiology of chondromalacia patellae. J Bone Joint Surg Br 1961;43-B:752-757

20 Chao EY, Neluheni EV, Hsu RW, Paley D. Biomechanics of malalignment. Orthop Clin North Am 1994;25(03):379-386

21 Hsu RW, Himeno S, Coventry MB, Chao EY. Normal axial alignment of the lower extremity and load-bearing distribution at the knee. Clin Orthop Relat Res 1990;(255):215-227

22 Cooke TD, Li J, Scudamore RA. Radiographic assessment of bony contributions to knee deformity. Orthop Clin North Am 1994;25 (03):387-393

23 Moreland JR, Bassett LW, Hanker GJ. Radiographic analysis of the axial alignment of the lower extremity. J Bone Joint Surg Am 1987;69(05):745-749

24 Paley D, Tetsworth K. Mechanical axis deviation of the lower limbs. Preoperative planning of uniapical angular deformities of the tibia or femur. Clin Orthop Relat Res 1992;(280):48-64

25 Fabricant PD, Camara JM, Rozbruch SR. Femoral deformity planning: intentional placement of the apex of deformity. Orthopedics 2013;36(05):e533-e537

26 Gaasbeek RD, Sonneveld H, van Heerwaarden RJ, Jacobs WC, Wymenga AB. Distal tuberosity osteotomy in open wedge high tibial osteotomy can prevent patella infera: a new technique. Knee 2004;11(06):457-461

27 Bito H, Takeuchi R, Kumagai K, et al. Opening wedge high tibial osteotomy affects both the lateral patellar tilt and patellar height. Knee Surg Sports Traumatol Arthrosc 2010;18(07):955-960

28 Pape D, Lorbach O, Schmitz C, et al. Effect of a biplanar osteotomy on primary stability following high tibial osteotomy: a biomechanical cadaver study. Knee Surg Sports Traumatol Arthrosc 2010;18(02):204-211

29 Benjamin A. Double osteotomy for the painful knee in rheumatoid arthritis and osteoarthritis. J Bone Joint Surg $\mathrm{Br}$ 1969;51(04):694-699 
30 Iveson JM, Longton EB, Wright V. Comparative study of tibial (single) and tibiofemoral (double) osteotomy for osteoarthrosis and rheumatoid arthritis. Ann Rheum Dis 1977;36(04):319-326

31 Schüller HM, van Dijk CN, Fidler MW. Poor results of double osteotomy for the rheumatoid knee. Acta Orthop Scand 1987; 58(03):253-255

32 Babis GC, An KN, Chao EY, Rand JA, Sim FH. Double level osteotomy of the knee: a method to retain joint-line obliquity. Clinical results. J Bone Joint Surg Am 2002;84-A(08):1380-1388

33 Saragaglia D, Mercier N, Colle PE. Computer-assisted osteotomies for genu varum deformity: which osteotomy for which varus? Int Orthop 2010;34(02):185-190

34 Saragaglia D, Blaysat M, Mercier N, Grimaldi M. Results of forty two computer-assisted double level osteotomies for severe genu varum deformity. Int Orthop 2012;36(05):999-1003

35 Saragaglia D, Chedal-Bornu B, Rouchy RC, Rubens-Duval B, Mader R, Pailhé R. Role of computer-assisted surgery in osteotomies around the knee. Knee Surg Sports Traumatol Arthrosc 2016;24(11):3387-3395

36 Lee YG, Park W, Kim SH, et al. A case of rhabdomyolysis associated with use of a pneumatic tourniquet during arthroscopic knee surgery. Korean J Intern Med 2010;25(01):105-109

37 Türkmen İ, Esenkaya İ, Unay K, Akçal MA. Rhabdomyolysis after tourniquet use in proximal tibial osteotomy: a case report and review of the literature. Acta Orthop Traumatol Turc 2015;49 (03):338-341

38 Melvin JS, Stryker LS, Sierra RJ. Tranexamic Acid in Hip and Knee Arthroplasty. J Am Acad Orthop Surg 2015;23(12):732-740

39 Xie J, Ma J, Yao H, Yue C, Pei F. Multiple Boluses of Intravenous Tranexamic Acid to Reduce Hidden Blood Loss After Primary Total Knee Arthroplasty Without Tourniquet: A Randomized Clinical Trial. J Arthroplasty 2016;31(11):2458-2464

40 Sun Q Yu X, Wu J, Ge W, Cai M, Li S. Efficacy of a Single Dose and an Additional Dose of Tranexamic Acid in Reduction of Blood Loss in Total Knee Arthroplasty. J Arthroplasty 2017;32(07): 2108-2112
41 Jung TM, Reinhardt C, Scheffler SU, Weiler A. Stress radiography to measure posterior cruciate ligament insufficiency: a comparison of five different techniques. Knee Surg Sports Traumatol Arthrosc 2006;14(11):1116-1121

42 Yu JS, Salonen DC, Hodler J, Haghighi P, Trudell D, Resnick D. Posterolateral aspect of the knee: improved MR imaging with a coronal oblique technique. Radiology 1996;198(01):199-204

43 LaPrade RF, Gilbert TJ, Bollom TS, Wentorf F, Chaljub G. The magnetic resonance imaging appearance of individual structures of the posterolateral knee. A prospective study of normal knees and knees with surgically verified grade III injuries. Am J Sports Med 2000;28(02):191-199

44 Badhe NP, Forster IW. High tibial osteotomy in knee instability: the rationale of treatment and early results. Knee Surg Sports Traumatol Arthrosc 2002;10(01):38-43

45 Arthur A, LaPrade RF, Agel J. Proximal tibial opening wedge osteotomy as the initial treatment for chronic posterolateral corner deficiency in the varus knee: a prospective clinical study. Am J Sports Med 2007;35(11):1844-1850

46 Noyes FR, Barber-Westin SD, Hewett TE. High tibial osteotomy and ligament reconstruction for varus angulated anterior cruciate ligament-deficient knees. Am J Sports Med 2000;28(03):282-296

47 Phisitkul P, Wolf BR, Amendola A. Role of high tibial and distal femoral osteotomies in the treatment of lateral-posterolateral and medial instabilities of the knee. Sports Med Arthrosc Rev 2006;14(02):96-104

48 Naudie DD, Amendola A, Fowler PJ. Opening wedge high tibial osteotomy for symptomatic hyperextension-varus thrust. Am J Sports Med 2004;32(01):60-70

49 Savarese E, Bisicchia S, Romeo R, Amendola A. Role of high tibial osteotomy in chronic injuries of posterior cruciate ligament and posterolateral corner. J Orthop Traumatol 2011; 12(01):1-17

50 LaPrade RF, Morgan PM, Wentorf FA, Johansen S, Engebretsen L. The anatomy of the posterior aspect of the knee. An anatomic study. J Bone Joint Surg Am 2007;89(04):758-764 